\title{
GLOBULAR CLUSTER AGES: ARE THEY CONVERGING?
}

\author{
DON A. VANDENBERG \\ Dept. of Physics \& Astronomy, University of Victoria \\ P.O. Box 3055, Victoria, B.C., Canada V8W $3 P 6$
}

\begin{abstract}
Since the early 1970's, the consensus "best estimate" of the age of the Galactic globular cluster (GC) system has been 15-16 \pm 3 Gyr. However, a number of recent studies, which are briefly reviewed herein, have suggested that this estimate is too high by $\gtrsim 15 \%$. Based on a consideration of new stellar models and the latest developments concerning the cluster distance scale, the present paper lends support to the notion that the first globulars began to form in the Galaxy 13-14 Gyr ago. In addition, our analysis of absolute GC ages adds to the mounting evidence that the dispersion in age among clusters having similar metallicities is probably quite small. As a consequence, age can hardly be the primary factor causing the wide diversity in their horizontal-branch morphologies.
\end{abstract}

\section{Introduction}

In a reasonably comprehensive review, VandenBerg, Bolte, \& Stetson (1996; VBS96) concluded that the most metal-deficient (presumably the oldest) globular clusters in the Galaxy have ages of $15_{+5}^{-3} \mathrm{Gyr}$, where the estimated uncertainties were considered to be approximate $2 \sigma$ limits. Virtually the same conclusion was reached by Chaboyer et al. (1996a) from a detailed Monte Carlo analysis of isochrone fits to observed C-M diagrams. However, a number of more recent investigations (Salaris, Degl'Innocenti, \& Weiss 1996; Jimenez et al. 1996; and D'Antona, Caloi, \& Mazzitelli 1997) have favored ages of $\lesssim 13 \mathrm{Gyr}$ for the oldest clusters and, moreover, they have suggested that a further reduction of 1-2 Gyr is well within the realm of possibility. Given the far-reaching implications for cosmology of such "young" ages, it is clearly important to examine how they were obtained and to assess their reliability.

T.R. Bedding et al. (eds.),

Fundamental Stellar Properties: The Interaction between Observation and Theory, 439-448.

(C) 1997 IAU. Printed in the Netherlands. 
Salaris et al. (1996) have remarked that, if the Buser \& Kurucz (1978, 1992; hereafter BK78, BK92) bolometric corrections are used to convert luminosity to $M_{V}$, instead of those tabulated by VandenBerg \& Bell (1985; VB85), about a $2 \mathrm{Gyr}$ reduction in the predicted ages for the most metalpoor globulars will result. On closer inspection, this assertion is not entirely correct because, in a relative sense, the $\mathrm{BK} 92 B C_{V}$ 's agree very well with those reported by VB85: their zero points do differ, but if both scales are normalized to some preferred value for the Sun, then the systematic variation of the $B C_{V}$ values with $T_{\text {eff }}$, gravity, or $[\mathrm{Fe} / \mathrm{H}]$ is small. (This should not be too suprising given that both studies used essentially the same model atmospheres.) Be that as it may, there are systematic differences between the BK78 $B C_{V}$ 's, on the one hand, and those given by VB85 and BK92, on the other. Furthermore, the BK78 bolometric corrections appear to be in better agreement with both the Kurucz (1992) scale and the latest predictions by R. Bell (1996) in the sense that, at temperatures and gravities characteristic of turnoff stars, all three predict a much larger variation in $B C_{V}$ as a function of $[\mathrm{Fe} / \mathrm{H}]$ than one obtains from either VB85 or BK92 (see VandenBerg 1997a). This dependence is such as to imply a decrease in GC ages amounting to roughly $\delta($ age $)=0.75[\mathrm{Fe} / \mathrm{H}] \mathrm{Gyr}$ compared with estimates based on VB85 or BK92 $B C_{V}$ 's. Since the recent Kurucz and Bell bolometric corrections are based on improved model atmospheres, they should be superior to earlier predictions; consequently, the VBS96 estimate of $15 \mathrm{Gyr}$ for the oldest GCs is arguably too high by $\sim 1.5 \mathrm{Gyr}$.

Jimenez et al. (1996; JTJMP) tried a new approach to derive GC ages. Specifically, they attempted to determine the masses (and hence the ages) of stars presently evolving on the giant branches of 8 globular clusters from the average mass on the horizontal branch (HB), the average massloss efficiency $\langle\eta\rangle$, where $\eta$ is the free parameter in the Reimers (1975) mass-loss formula, and its dispersion. To accomplish this, they adopted weighted averages of the cluster $[\mathrm{Fe} / \mathrm{H}], E(B-V)$, and $(m-M)_{V}$ values from the scientific literature along with the Kurucz (1992) color transformations. Published theoretical models were then used to infer the observed HB mass distributions - a risky procedure since the location of an HB star on the H-R diagram is known to be very sensitive to many parameters (cf. Tornambe 1988), and because the evolutionary state of a given HB star can often be ambiguous, thereby confusing the mass determination. Moreover, because masses tend to "pile up" at the red end, small errors in color $/ T_{\text {eff }}$ will necessarily translate to large errors in the estimated masses of red horizontal-branch stars in clusters possessing them.

By requiring agreement between the "observed" HB mass distribution in each GC and that predicted from numerical integrations of the mass loss along the upper RGB, $\langle\eta\rangle$ was determined. JTJMP appear to have 
either assumed or concluded from their analysis that the reddest star on an observed HB did not undergo any prior mass loss: they state (p. 938) that " $\langle\eta\rangle$ has been calculated from the value needed to reproduce both the point in the HB where $\eta=0$ and the mean HB mass". This is highly questionable given the growing evidence (e.g., see the review by Stetson, VandenBerg, \& Bolte 1996) against the hypothesis that age is the second parameter. For instance, M3 and M13 appear to be nearly coeval (see Ferraro et al. 1997); hence, for some yet unknown reason (differences in stellar rotation?), the HB stars in M13, which are almost entirely to the blue of the instability strip, must have undergone much more extensive mass loss than their counterparts in M3, which extend to very red colors. According to JTJMP, variations in HB morphology are due to differences in age.

Some other concerns with the JTJMP paper include the fact that their adopted cluster distances and derived ages are not consistent with turnoff age-luminosity relations. For instance, their derived age of $13.2 \mathrm{Gyr}$ for M92 can only be obtained from an isochrone fit to the turnoff photometry if $(m-M)_{V}>14.65$ (see $\left.\S 2\right)$ - assuming best estimates for the chemical abundances - whereas JTJMP adopted $(m-M)_{V}=14.45$ (for which the turnoff age has to be $>16 \mathrm{Gyr}$ ). They suggest that a precise age cannot be accurately determined from the turnoff, even if the cluster distance were known, in part because of complications due to the mixing-length parameter. Granted, it is frequently quite difficult to define the turnoff point in an observed C-M diagram to within $\pm 0.1 \mathrm{mag}$; however, in practice (see $\S 2$ ), it is from the coincidence of the predicted and observed subgiant branch that the cluster age is inferred. The associated internal error is certainly $< \pm 0.5$ Gyr (cf. Chaboyer et al. 1996b). Both the distance scale problem and the small numbers of upper RGB stars in their samples (as few as 25 within 2 mag of the RGB tip) are further cause for skepticism. The approach used by JTJMP to derive GC ages, and their results, appear to be much more uncertain than they have acknowledged ${ }^{1}$.

Finally, D'Antona et al. (1997) have suggested that the most metalpoor GCs may be as young as 12 Gyr. The 2-3 Gyr reduction in age from canonical estimates is due to two factors: (1) the use of the Canuto \& Mazzitelli (1991) theory of convection in their calculations instead of the

\footnotetext{
${ }^{1}$ More recently, Jimenez \& Padoan (1996) have obtained an age of 16.4 Gyr for M68 from an analysis of its luminosity function, on the assumption of nearly the same distance modulus that was adopted by JTJMP, who found an age of 12.7 Gyr for this cluster. No explanation was offered as to the cause of the discrepant results. In this particular case, the adopted distance, $(m-M)_{V}=15.3$, simply cannot be reconciled with an age as high as 16 Gyr (see §3). The Jimenez-Padoan study is somehow flawed, though perhaps only in something as basic as their assumption that the relative lifetimes of stars in different evolutionary phases are accurately predicted by standard models - for which there is some evidence to the contrary (Bolte 1994, and references therein).
} 
usual mixing-length theory, and (2) the adoption of increased distances to the globulars to be consistent with the prediction of their HB models. In an earlier paper, Mazzitelli, D'Antona, \& Caloi (1995) demonstrated quite convincingly that turnoff age-luminosity relations do depend on how convection is treated, particularly those for low-metallicity stars that have very thin convective envelopes. VBS96 have argued that this uncertainty is alleviated to some extent by inferring cluster ages from the luminosities of subgiant-branch stars, which have much deeper surface convection zones. Even so, it remains a real possibility that the treatment of convection can affect GC age determinations at the $\sim \pm 1$ Gyr level.

As far as predicted HB luminosities are concerned, it has not been possible to reach a consensus, neither theoretically nor empirically (see VBS96), on what the true relation is between $M_{V}(\mathrm{HB})$ and $[\mathrm{Fe} / \mathrm{H}]$. The horizontalbranch models that D'Antona et al. have used in their analysis appear to be brighter than those used in this investigation $(\S 2)$ because they have somewhat larger helium core masses, but the cause of the differences in this quantity is presently unknown. Fortunately, Hipparcos data (see the previous paper in these proceedings), the determination of white dwarf distances to GCs (e.g., Renzini et al. 1996), and the observation of HB stars in M31 globular clusters (e.g., Fusi Pecci et al. 1996) promise to resolve these issues. First indications are (see below) that these constraints favor fainter HB luminosities (and older GC ages) than D'Antona et al. have advocated.

\section{Absolute Globular Cluster Ages}

VandenBerg et al. (1997) have computed an extensive new grid of evolutionary tracks and isochrones for ages from 8 to $18 \mathrm{Gyr}$, as well as fullyconsistent zero-age horizontal-branch (ZAHB) loci, assuming $[\alpha / \mathrm{Fe}]=0.0$, 0.3 , and 0.6 for each of more than a dozen $[\mathrm{Fe} / \mathrm{H}]$ values between -2.3 and -0.3 . All $\alpha$ elements, including $\mathrm{O}, \mathrm{Ne}, \mathrm{Mg}, \mathrm{Si}, \mathrm{S}$, etc., were assumed to vary together in abundance, and OPAL opacities (cf. Rogers \& Iglesias 1992) were calculated for the adopted mixes. Furthermore, an improved equation of state allowing for Coulomb interactions and other non-ideal effects was employed, along with the rates for H-burning reactions described by Bahcall $\&$ Pinsonneault (1992). The transformation of the models from the theoretical to various C-M planes was carried using an empirically-constrained set of color transformations (VandenBerg 1997b) and the Kurucz (1992) bolometric corrections, adjusted by a constant amount to ensure consistency between the adopted $M_{\mathrm{bol}}$ and observed $M_{V}$ values for the Sun.

The left-hand panel in Figure 1 shows that if the distance to M92 is set by matching the predicted and observed ZAHBs - yielding $(m-M)_{V}=$ 


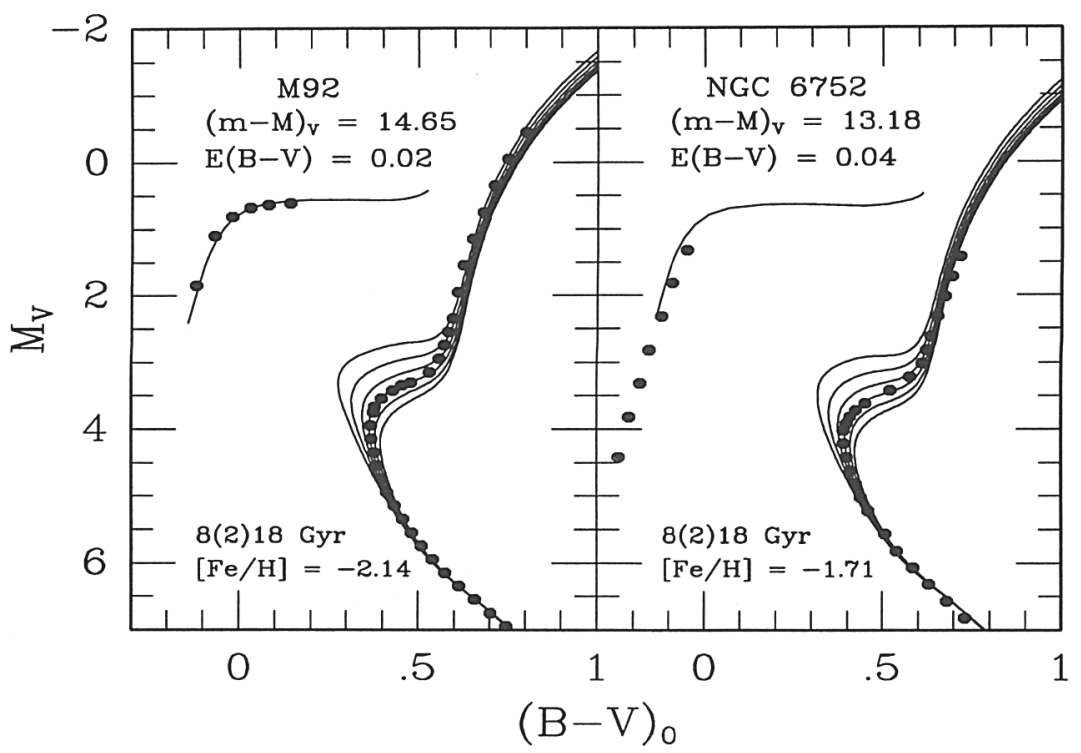

Figure 1. Comparisons of isochrones for the specified ages and $[\mathrm{Fe} / \mathrm{H}]$ values with fiducial sequences for M92 (Stetson \& Harris 1988, Bolte 1997) and NGC 6752 (as given by VBS90, based on photometry by Penny \& Dickens 1986) for the indicated reddenings and distances. The position of the blue ZAHB in NGC 6752 was derived from an eye-estimated fit to the lower bound of the observations plotted by Caloi et al. (1986; their Fig. 1). Small color adjustments $(<0.015 \mathrm{mag})$ were applied to the isochrones, as necessary, in order to ensure that the predicted and observed lower main sequences coincided. In this, and all other plots to follow $[\alpha / \mathrm{Fe}]=0.3$ has been assumed: see Carney (1996) and Ryan, Norris, \& Beers (1996) for justification of this choice.

14.65, which is identical to the value adopted by VBS96 from a consideration of the classical Population II subdwarfs - an age near 14 Gyr is obtained. (We emphasize that the same isochrones were used in both studies and that differences in the derived age are entirely due to the $B C_{V}$ scale revisions adopted here.) The right-hand panel indicates a very similar age for NGC 6752 on the assumption of the distance modulus that Renzini et al. (1996) have derived by fitting their $H S T$ observations of cluster white dwarfs to local white dwarf sequences. They suggest that this distance determination should be accurate to $\sim 10 \%$. Interestingly, in spite of having an extremely blue HB, NGC 6752 does not appear to have an extreme age.

From the close similarity of the M3 and NGC $6752 \mathrm{C}-\mathrm{M}$ diagrams between the lower main sequence and the lower RGB, VandenBerg, Bolte, \& Stetson (1990; VBS90) argued that the two clusters must be nearly coeval, despite their very different HB morphologies (also see Richer et al. 1996). And, indeed, when ZAHB luminosities are used to infer the distance to M3, an isochrone fit to the turnoff photometry indicates an age of 13-14 


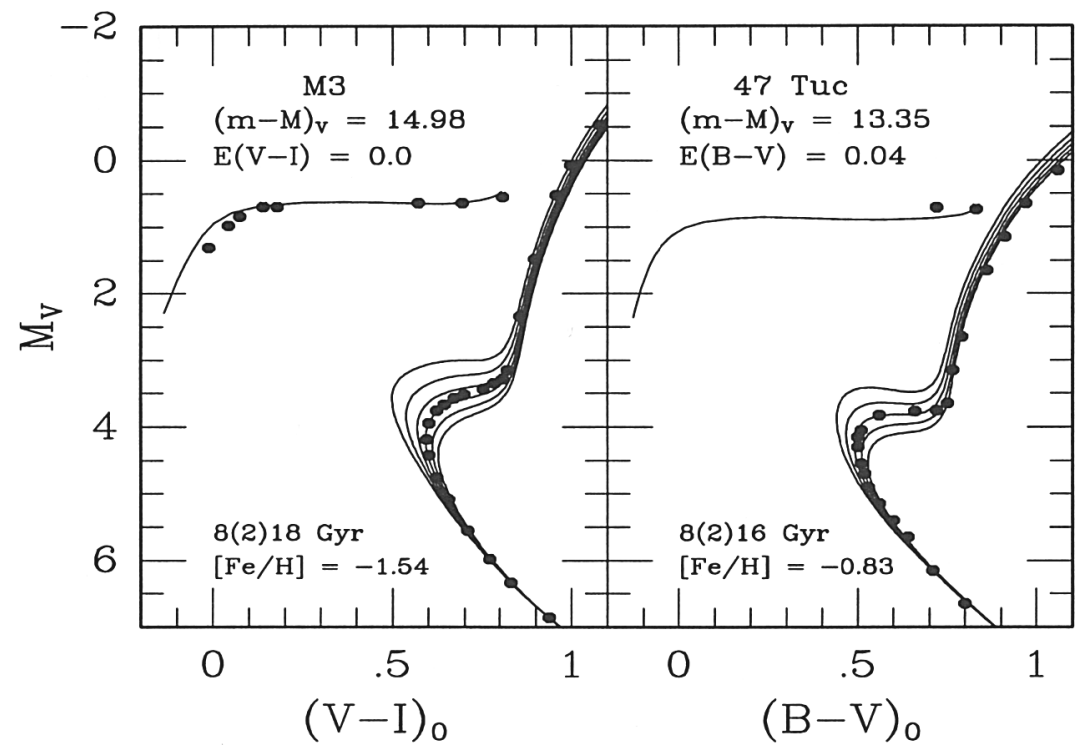

Figure 2. Similar to Fig. 1; in this case, isochrones for the specified ages and $[\mathrm{Fe} / \mathrm{H}]$ values are compared with fiducial sequences for M3 (Johnson \& Bolte 1997) and 47 Tucanae (Hesser et al. 1987).

Gyr (see the left-hand panel of Figure 2). The very encouraging agreement between the relative cluster ages as derived from a distance-independent method (VBS90) and from an evaluation of their absolute ages using different standard candles to determine the cluster distances suggests that the adopted moduli and the different approaches to the data are quite reliable. Although the uncertainties are still large enough not to preclude a 1-2 Gyr difference in age between M3 and NGC 6752, a variation of this size is at least a factor of 3 too small to explain the difference in their HB populations in terms of age. Thus the present analysis provides yet another (rather compelling) argument that age cannot be the second parameter. It is also worth mentioning that the relation between $M_{V}(\mathrm{HB})$ and $[\mathrm{Fe} / \mathrm{H}]$ predicted by VandenBerg et al. (1997), namely $M_{V}(\mathrm{HB})=0.19[\mathrm{Fe} / \mathrm{H}]+0.96$ (evaluated at $\log T_{\text {eff }} \approx 3.84$ and assuming $\left.[\alpha / \mathrm{Fe}]=0.3\right)$, agrees well with those predicted by other workers (e.g., Lee 1990) and that derived from $H S T$ observations of M31 globular clusters (Fusi Pecci et al. 1996). However, Baade-Wesselink and statistical parallax studies of field RR Lyraes appear to favor fainter HB luminosities (see the VBS96 review) and hence older ages for the globular clusters (e.g., see Carney 1996).

As illustrated in the right-hand panel of Fig. 2, a turnoff age of $\approx 12 \mathrm{Gyr}$ is predicted for 47 Tuc if its apparent distance modulus is 13.35 - and the cluster stars have $[\mathrm{Fe} / \mathrm{H}]=-0.83$ (e.g., Brown, Wallerstein, \& Oke 1990), $[\alpha / \mathrm{Fe}]=0.3$ (cf. Carney 1996), and $Y=0.24$ (Dorman, VandenBerg, \& 
Laskarides 1989). It may be recalled that Hesser et al. (1987) derived an age of $13.5 \mathrm{Gyr}$ for this cluster, assuming $(m-M)_{V}=13.40$ and isochrones for $[\mathrm{Fe} / \mathrm{H}]=-0.65,[\mathrm{O} / \mathrm{Fe}]=0.3$, and $Y=0.24$. While it may initially seem a bit disconcerting that a $1.5 \mathrm{Gyr}$ younger age is obtained on the assumption of a slightly reduced distance (which would normally imply an increased age), there is no inconsistency. The cumulative effect of the revisions to the $B C_{V}$ scale, the adoption of $[\alpha / \mathrm{Fe}]=0.3$ instead of $[0 / \mathrm{Fe}]$ $=0.3$, and the treatment of Coulomb interactions in the equation of state used by VandenBerg et al (1997) is to reduce the age by $\gtrsim 1.5 \mathrm{Gyr}$ at a given turnoff luminosity. Although Figs. 1 and 2, taken together, intimate that the Galactic GCs obey an age-metallicity relation, which they may well do, the sample of clusters considered here is obviously too small and the uncertainties still too large to allow one to regard these findings as more than suggestive.

\section{On the Relative Ages of M68 and M92}

VBS90 found that, when the C-M diagrams of M30, M68, and NGC 6397 were individually shifted both vertically and horizontally by the amounts needed to register them to the turnoff magnitude and color of M92, there was no perceptible difference in the location of their respective RGBs. Since a similar registration of theoretical isochrones relevant to these clusters indicated that the color difference between the turnoff and the lower giant branch changes at the rate $\Delta(B-V)_{\mathrm{TO}, \mathrm{RGB}} \approx-0.012 \mathrm{mag} / \mathrm{Gyr}, \mathrm{VBS} 90$ concluded that the 4 clusters were "extremely uniform in age, with no convincing evidence for differences as great as 0.5 Gyr". Richer et al. (1996) reached basically the same conclusion, using the same approach, for a much larger sample of very metal-poor GCs. In stark contrast with this, Chaboyer, Demarque, \& Sarajedini (1996c; CDS96) argued from measurements of the magnitude difference between the main-sequence turnoff and the $\mathrm{HB}, \Delta V_{\mathrm{TO}}^{\mathrm{HB}}$, that there is a range of $\gtrsim 5 \mathrm{Gyr}$ amongst these same clusters. Who is right?

In principle, the $\Delta(B-V)_{\text {TO,RGB }}$ method is capable of much higher precision largely for the reason that the turnoff to lower-RGB color difference can be determined much more accurately than $\Delta V_{\mathrm{TO}}^{\mathrm{HB}}$ (see the extensive discussion of the advantages and disadvantages of the two techniques given by Stetson et al. 1996). Because, by definition, a C-M diagram is vertical at the turnoff, the turnoff magnitude cannot be determined more precisely than about $\pm 0.05-0.1 \mathrm{mag}$, even from superb photometry. Since the rate at which $\Delta V_{\mathrm{TO}}^{\mathrm{HB}}$ changes with age is approximately $+0.072 \mathrm{mag} / \mathrm{Gyr}-$ the estimated slope of turnoff $M_{V}$ versus age relations at a representative age of 14 Gyr (VandenBerg et al. 1997) - the $\Delta V_{\mathrm{TO}}^{\mathrm{HB}}$ method as it has 


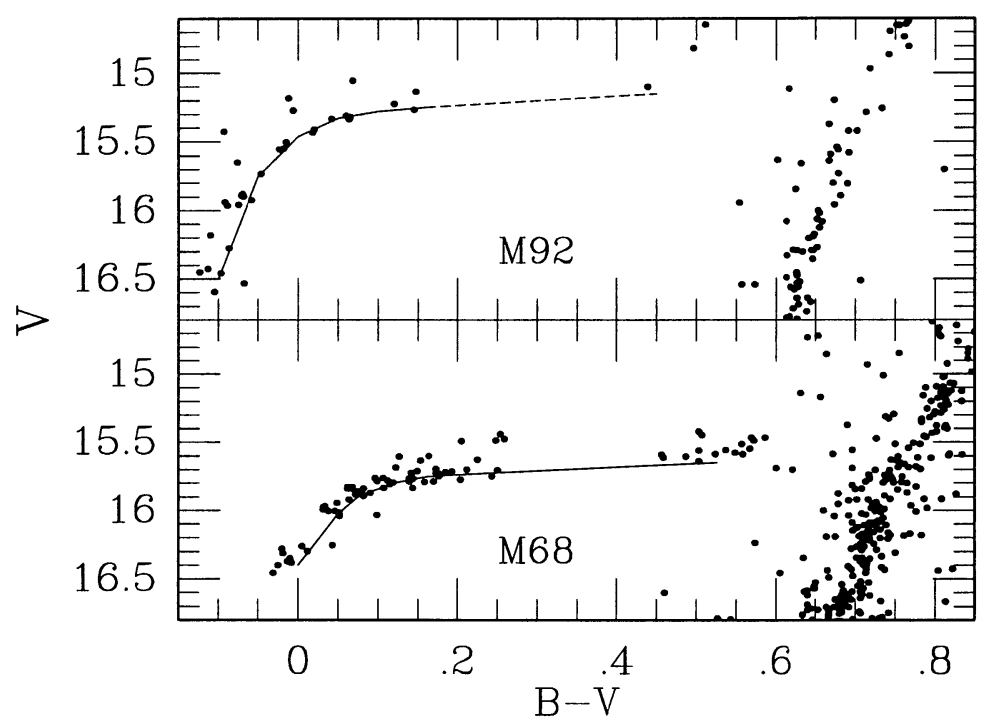

Figure 3. Plot of Bolte (1997) and Walker (1994) M92 and M68 photometry, respectively, for RGB and non-variable HB stars having $14.6 \leq V \leq 16.8$. The solid curves give eye-estimated ZAHB loci. The dashed curve extrapolates the M92 locus to redder colors: its slope is taken to be the same as that of the M68 ZAHB through the instability strip.

traditionally been used cannot reliably detect a difference in age smaller than $\sim 1$ Gyr. Furthermore, many GCs have few (or no) HB stars at the color of the turnoff, and even when they do, there could well be clusterto-cluster differences in the mean evolutionary state of those stars, further complicating the evaluation of the $\Delta V_{\mathrm{TO}}^{\mathrm{HB}}$ parameter.

According to CDS96, the $\Delta V_{\mathrm{TO}}^{\mathrm{HB}}$ values for M30, M68, NGC 6397, and $\mathrm{M} 92$ are $3.62 \pm 0.14,3.42 \pm 0.10,3.74 \pm 0.12$, and $3.74 \pm 0.14$, respectively. Only in the case of M68 can one plausibly argue for a difference in age. But do the $\Delta V_{\mathrm{TO}}^{\mathrm{HB}}$ values for M68 and M92 really differ by $0.32 \mathrm{mag}$ ? Although based on relatively few stars, McClure et al. (1987) found no obvious difference in the location of the HB populations of M68 and M15 when the turnoffs of the two clusters were aligned: CDS96 give $\Delta V_{\mathrm{TO}}^{\mathrm{HB}}=$ $3.63 \pm 0.16$ for M15. Subsequently, Carney, Storm, \& Jones (1992, see their Fig. 22) argued that there was no difference in the relative ages of M68 and M92 using either the $\Delta V_{\mathrm{TO}}^{\mathrm{HB}}$ or $\Delta(B-V)_{\mathrm{TO}, \mathrm{RGB}}$ methods. And, if one defines ZAHB loci for M68 and M92 from the latest available photometry (see Figure 3), and compares them once the cluster turnoffs have been registered to one another (thereby removing most of the uncertainty in the definition of $V_{\mathrm{TO}}$ ), the result is Figure 4. The difference in the $\Delta V_{\mathrm{TO}}^{\mathrm{HB}}$ values for the two clusters is only $0.08 \mathrm{mag}$, suggesting rather similar ages. Perhaps the greatest concern with this analysis is that the data are taken 


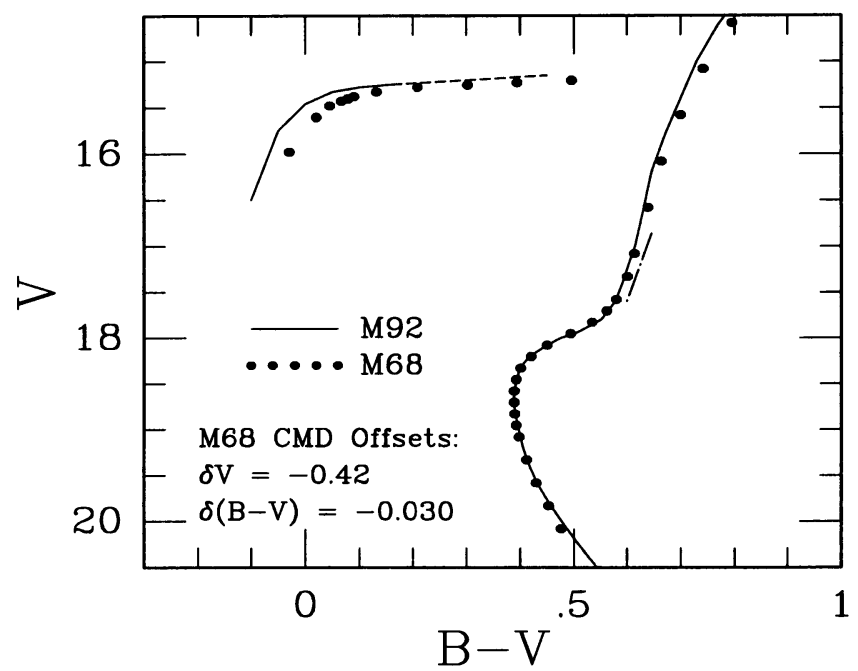

Figure 4. Using the indicated offsets and the ZAHB loci from Fig. 3, the fiducial sequences for M68 (McClure et al. 1987) and M92 (Stetson \& Harris 1988) are intercompared. The dot-dashed line shows where the lower RGB of M68 would be located if this cluster were 2 Gyr younger than M92.

from several sources, inviting the possibility that small differences exist in the respective photometric zero-points which could have some ramifications for the measured $\Delta V_{\mathrm{TO}}^{\mathrm{HB}}$ values. (This is a potential problem for much of the CDS96 dataset as well.) But, until more definitive studies are carried out, we conclude that there is no inconsistency in the results of the $\Delta V_{\mathrm{TO}}^{\mathrm{HB}}$ and $\Delta(B-V)_{\text {TO,RGB }}$ methods as applied to M68 and M92. Indeed, relative GC age estimates based on the color-difference technique are arguably much more secure than those based on the $\Delta V_{\mathrm{TO}}^{\mathrm{HB}}$ method.

\section{Summary}

Due to steady improvements to the stellar models and tightening constraints on the cluster distances, some convergence of GC ages is certainly occurring. This study suggests that (1) the most metal-poor GCs formed about $14 \mathrm{Gyr}$ ago, (2) the globulars obey an age-metallicity relation with $[\mathrm{Fe} / \mathrm{H}] \sim-0.8$ clusters like 47 Tuc being about 2 Gyr younger than very metal-deficient systems, (3) M3 and NGC 6752 have similar ages despite huge differences in their HB morphologies, and (4) M68 and M92 are close to being coeval in spite of recent claims to the contrary. (The absolute ages derived here should probably be reduced by $\sim 1$ Gyr to allow for the effects of He diffusion, which was not treated in the present models.) 
A Killam Research Fellowship and the support of an N.S.E.R.C. operating grant are gratefully acknowledged. I thank M. Bolte for permission to use his M3 and M92 photometry prior to publication.

\section{References}

Bahcall, J.N. \& Pinsonneault, M.H. 1992, Rev.Mod.Phys., 64, 885

Bell, R.A. 1996, private communication

Bolte, M. 1994, Ap.J., 431, 223

Bolte, M. 1997, private communication

Brown, J.A., Wallerstein, G., \& Oke, J.B. 1990, A.J., 100, 1561

Buser, R. \& Kurucz, R.L. 1978, Astron.Astrophys., 70, 555 (BK78)

Buser, R. \& Kurucz, R.L. 1992, Astron.Astrophys., 264, 557 (BK92)

Caloi, V., Castellani, V., Danziger, J., et al. 1986, M.N.R.A.S., 222, 55

Canuto, V.M. \& Mazzitelli, I. 1991, Ap.J., 370, 295

Carney, B.W. 1996, P.A.S.P., 108, 900

Carney, B.W., Storm, J., \& Jones, R.V. 1992, Ap.J., 386, 663

Chaboyer, B., Demarque, P., Kernan, P.J., \& Krauss, L.M. 1996a, Science, 271, 957

Chaboyer, B., Demarque, P., Kernan, P.J., et al. 1996b, M.N.R.A.S., 283, 683

Chaboyer, B., Demarque, P., \& Sarajedini, A. 1996c, Ap.J., 459, 558 (CDS96)

D'Antona, F., Caloi, V., \& Mazzitelli, I. 1997, Astron.Astrophys., in press

Dorman, B., VandenBerg, D.A., \& Laskarides, P.G. 1989, Ap.J., 343, 750

Ferraro, F.R., Paltrinieri, B., Fusi Pecci, F., et al. 1997, Ap.J.Lett., submitted

Fusi Pecci, F., Buonanno, R., Cacciari, C., et al. 1996, A.J., 112, 1461

Hesser, J.E., Harris, W.E., VandenBerg, D.A., et al. 1987, P.A.S.P., 99, 739

Jimenez, R. \& Padoan, P. 1996, Ap.J.Lett., 463, L17

Jimenez, R., Thejll, P., Jorgensen, U.G., MacDonald, J., \& Pagel, B. 1996, M.N.R.A.S., 282, 926 (JTJMP)

Johnson, J. \& Bolte, M. 1997, in preparation

Kurucz, R.L. 1992, Solar Abundance Model Atmospheres (CD-ROM 19)

Lee, Y.-W. 1990, Ap.J., 363, 159

Mazzitelli, I., D'Antona, F., \& Caloi, V. 1995, Astron.Astrophys., 302, 382

McClure, R.D., VandenBerg, D.A., Bell, R.A., et al. 1987, A.J., 93, 1144

Penny, A.J. \& Dickens, R.J. 1986, M.N.R.A.S., 220, 845

Reimers, D. 1975, Mem.Soc.Roy.Sci. Liège, 8, 369

Renzini, A., Bragaglia, A., Ferraro, F.R., et al. 1996, Ap.J.Lett., 465, L23

Richer, H.B., Harris, W.E., Fahlman, G.G., et al. 1996, Ap.J., 463, 602

Rogers, F.J. \& Iglesias, C.A. 1992, Ap.J.Suppl., 79, 507

Ryan, S.G., Norris, J.E., \& Beers, T.C. 1996, Ap.J., 471, 254

Salaris, M., Degl'Innocenti, S., \& Weiss, A. 1996, preprint

Stetson, P.B. \& Harris, W.E. 1988, A.J., 96, 909

Stetson, P.B., VandenBerg, D.A., \& Bolte, M. 1996, P.A.S.P., 108, 560

Tornambé, A. 1987, in ESO Workshop on Stellar Evolution and Dynamics in the Outer Halo of the Galaxy, ed. M. Azzopardi \& F. Matteucci (Garching: ESO), p. 307

VandenBerg, D.A. 1997a, in Advances in Stellar Evolution, ed. R.T. Rood \& A. Renzini (Cambridge: Cambridge U. Press), in press

VandenBerg, D.A. 1997b, in preparation

VandenBerg, D.A. \& Bell, R.A. 1985, Ap.J.Suppl., 58, 561 (VB85)

VandenBerg, D.A., Bolte, M., \& Stetson, P.B. 1990, A.J., 100, 445 (VBS90)

VandenBerg, D.A., Bolte, M., \& Stetson, P.B. 1996, Ann.Rev.Astron.Astrophys., 34, 461 (VBS96)

VandenBerg, D.A., Swenson, F.J., Rogers, F.J., Iglesias, C.A., \& Alexander, D.R. 1997, in preparation

Walker, A.R. 1994, A.J., 108, 555 